\section{Comparisons of intervals between subjective numbers*}

\author{
STANLEY J. RULE \\ University of Alberta, Edmonton, Alberta, Canada
}

Fifty Ss compared the subjective magnitudes of adjacent, objectively equal intervals between numbers by the method of triads. The numbers investigated were the integers from 1 to 10 . For every comparison, the interval between the larger integers was more frequently judged closer, which supported previous findings that, for numbers used in magnitude estimation, subjective number is a negatively accelerated function of objective number. The nature of the psychological and physical variables in a number psychophysical function was discussed.

Magnitude estimation entails a conjoining of two psychophysical relationships, one between sense impressions and physical stimuli and one between subjective number and objective number (Attneave, 1962). Until very recently, the latter psychophysical relationship has received little attention. Usually it has been tacitly assumed that the number function was linear. However, a series of studies using magnitude estimation of paired stimuli has provided evidence that the psychophysical relationship for number is a power function (Curtis, 1970; Curtis, Attneave, \& Harrington, 1968; Curtis \& Fox, 1969; Rule, Curtis, \& Markley, 1970). Estimates of the exponent ranged from 0.6 to 0.9 , indicating that subjective number was a negatively accelerated function of objective number. The Ss were required to estimate either differences in stimulus magnitudes or sums of stimulus magnitudes. An equation based on a two-stage model proposed by Attneave (1962) was fitted to the data. The analysis yielded two exponents, one from a sensory function and the other the reciprocal of an exponent for number. The product of the two exponents provided a good approximation to the exponent from magnitude estimation of single stimuli, a result consistent with the two-stage model.

In another approach to investigating subjective number, Rule (1969) obtained a discriminability scale of number. Rather than requiring Ss to give numerical responses to each stimulus, Ss were presented with a circle and a number and asked to judge which had the greater magnitude.

\footnotetext{
* This research was supported by Grant APA-151 from the National Research Council of Canada. The paper was written while the author was on leave at the Department of Mathematical Psychology, University of Nijmegen. The Netherlands. The assistance of Marian Kinkaide in the collection and analysis of the data is collection and analysis
gratefully acknowledged.
}

Scale values for number were obtained from a Thurstone-type discriminability model. The scale values were found to be a negatively accelerated function of objective number. Initially, the function was described as logarithmic, but later Rule et al (1970) noted that the data showed less curvature than a logarithmic function and indicated that a power function with an exponent of 0.4 provided a better approximation to the data. This finding was confirmed in a replication in which numbers were compared with weights (Rule, 1971). The exponent was 0.5 .

The present study employed a more direct approach for investigating subjective number. Ss were asked to compare intervals between numbers. Such an approach provides information on the general form of the number function. If it is linear, the intervals should remain constant. Alternatively, if subjective number is a negatively accelerated function of objective number, subjective intervals should come progressively closer.

\section{METHOD}

The Ss were 50 students enrolled in an introductory course in psychology. Participation in psychological experiments was a required part of the course work.

There were two stages to the procedure. First, Ss were provided with experience in using numbers in making magnitude estimations, and second, they were asked to compare intervals between numbers. It was the latter task which was of experimental interest.

The stimuli for magnitude estimation were 12 circles prepared on $35-\mathrm{mm}$ slides. The areas of the projected circles ranged in equal logarithmic steps from 1.37 to $303.56 \mathrm{~cm}^{2}$. The Ss were instructed to assign the value 10 to a standard circle $\left(26.07 \mathrm{~cm}^{2}\right)$ and to assign to subsequent circles numbers proportional to the $S$ 's subjective impression of their area. The series of 12 circles was presented two times in a random order.

After the magnitude estimation task was completed, Ss were provided with some general information on psychophysical scaling followed by instructions for comparing intervals between numbers. The essential parts of the information and instructions were:

"This research is in an area known as psychophysical scaling. In this area we are interested in the relation between physical measures of stimuli and subjective or psychological measures. People's subjective impression of stimuli do not necessarily correspond exactly to physical measurements. For example, a person's impression of the loudness of a sound does not correspond directly to its intensity. A person's judgment of loudness depends in part on the pitch of the sound. Many other variables, as well as the physical properties of the stimulus, enter into the judgment. One important variable which influences a person's judgment is the way he uses numbers in making his estimates. This depends on his subjective impression of numbers. One's subjective impression of numbers differs from the way we think of numbers when doing arithmetic.

"I am going to ask some questions about your subjective impression of numbers. Do not determine the answer by doing any arithmetic, but answer the questions by giving your own impression. The questions will be of this form. I will say a number, such as 50 , and will ask you which is closer to

Table 1

Proportion of Closer Judgments of the Larger $V$ ariable Integer

\begin{tabular}{cllllllll}
\hline & \multicolumn{7}{c}{ Larger Variable Integer } \\
\cline { 2 - 9 } Integer & $\mathbf{3}$ & 4 & $\mathbf{5}$ & $\mathbf{6}$ & $\mathbf{7}$ & $\mathbf{8}$ & 9 & $\mathbf{1 0}$ \\
\hline 2 & .51 & & & & & & & \\
3 & & .54 & .62 & & & & & \\
4 & & & .73 & .69 & .64 & & & \\
5 & & & & .63 & .73 & .67 & .74 & .73 \\
6 & & & & & .63 & .74 & .71 & .77 \\
7 & & & & & & .77 & .70 & .76 \\
8 & & & & & & & .65 & .77 \\
9 & & & & & & & & \\
\hline
\end{tabular}


it, 25 or 75 . It is obvious that the two numbers are arithmetically an equal distance from 50. But $\mathrm{I}$ am interested in your subjective impression. Your own impression may be that 50 seems closer to 25 , or it may be that 50 seems closer to 75. That would be your own subjective impression."

It is important to note that the term "closer" was defined by the example as a smaller absolute difference. The numbers investigated were the integers from 1 to 10 . They were presented verbally in a question of the form: "Is $\mathrm{N}_{\mathrm{s}}$ closer to $\mathrm{N}_{\mathrm{i}}$ or $\mathrm{N}_{\mathrm{j}}$ ?" (e.g., "Is 5 closer to 3 or 7?"). The standard integer, $\mathrm{N}_{\mathrm{s}}$ (e.g., 5 ), was always the midpoint of the variable integers, $N_{i}$ and $N_{j}$ (e.g., 3 and 7 ), so that the absolute difference between the standard and each variable integer was objectively equal. Hence, the 20 possible triads so constructed consisted of 8 with a difference between the standard and each variable integer of one unit, 6 with a difference of two units, 4 with a difference of three units, and 2 with a difference of four units. Such a series of triads was presented two times, with the order of presentation randomized independently for each $S$. Order of appearance of the two variable integers in each triad was counterbalanced such that the larger variable integer appeared first on one-half of the triads on their first presentation, and the order was reversed on the second presentation.

\section{RESULTS}

For each triad the proportion of responses in which the larger variable integer was judged closer to the standard integer is presented in Table 1. All proportions were greater than 0.50 , indicating that adjacent intervals were successively closer. Also recorded were responses to the example question comparing 50 with 25 and 75. Seventy percent of the Ss selected 75 as closer to 50 , which provides weak evidence that the results may hold beyond the range of numbers investigated.

Responses from each $S$ were summed over triads. Forty-two of the 50 Ss more frequently judged the higher variable integer as closer to the standard, a frequency reliably greater than one-half of the Ss $(z=4.67$, $\mathrm{p}<.01$ ). This result indicates that the impression of progressively closer integers held for most Ss. A comment volunteered by a $S$ at the end of the experimental session well summarizes the results: "The numbers seem to come closer together like railroad ties in the distance."

\section{DISCUSSION}

The present results indicated that for the integers investigated, those from 1 to 10, subjective impression of numbers used in magnitude estimation was a negatively accelerated function of objective number. Such a relation excludes a linear function but does not distinguish between a logarithmic function and a power function with an exponent less than 1.0. However, evidence from magnitude estimation of paired stimuli (Curtis, 1970; Curtis et al, 1968; Curtis \& Fox, 1969; Rule et al, 1970) and from discriminability scaling of number (Rule, 1969), as analyzed by Rule et al (1970) and replicated by Rule (1971), indicate a power function for subjective number.

In the present data there is an inconsistency with a power function (and a logarithmic function as well). The proportions of 0.51 and 0.54 for the triads of integers 1, 2, and 3 and of 2,3 , and 4 were much smaller than the other triads which have an interval of 1.0 between the standard and variable integers. A larger proportion would be expected. This inconsistency may indicate systematic deviations from a power function or biases introduced by the experimental procedure. Because comparisons of the proportions involve third-order differences between subjective impressions of the stimuli, which demand highly stable data, this inconsistency should be interpreted with caution.

The variables entering into a psychophysical function of number differ from those in the usual sensory function. The physical variable, objective number, is an abstraction based on logic. It is the abstract quantity represented by a number name. The psychological measure is of a conceptual process, developed through learning, rather than a sensory one. It is not the visual or auditory impression of numerals which is measured, but it is the conception of abstract quantities represented by the numerals. The number function may vary for different tasks, depending on S's past experience with the particular use of numbers required by the task. A psychophysical function of numbers for magnitude estimation may differ from one for mental arithmetic. The relation between subjective and objective number for mental arithmetic is necessarily one of identity since the $S$ relies on well-learned arithmetic tables stored in memory. When estimating stimulus magnitudes, the $\mathrm{S}$ has no such tables available. Furthermore, for different judgment tasks the number function may vary. Evidence was provided by Curtis (1970) and Curtis and Fox (1969) that the psychophysical function for numbers used as category labels differs from that of numbers used in magnitude estimation.

For numbers used in magnitude estimation, what past experience might lead to a power function with an exponent less than 1.0? One possibility is that Ss' impressions of numbers stem from experience with two relations between numbers used in measurement, absolute change and relative change. For example, Ss have experienced increases from 10 to 12 and from 20 to 22 as an absolute increase of two units and as relative increases of $20 \%$ and $10 \%$, respectively. Subjective impression of number based on absolute change would produce a linear function, whereas one based on relative change would yield a logarithmic function. Experience with both relations may produce an intermediate function, one which is negatively accelerated but with less curvature than a logarithmic function. Such a relationship would be either a power function or one closely approximated by a power function.

\section{REFERENCES}

ATTNEAVE, F. Pexception and related areas. In S. Koch (Ed.), Psychology: A study of a science. Vol 4. New York: McGraw-Hill, 1962. Pp. 619-659.

CURTIS, D. W. Magnitude estimations and category judgments of brightness and brightness intervals: A two-stage interpretation. Joumal of Experimental Psychology, 1970, 83, 201-208.

CURTIS, D. W., ATTNEAVE, F., \& HARRINGTON, T. L. A test of a two-stage model of magnitude judgment. Perception \& Psychophysics, 1968, 3, 25-31.

CURTIS, D, w, \& FOX, B. E. Direct quantitative judgments of sums and a two-stage model for psychophysical judgments. Perception \& Psychophysics, $1969,5,89-93$.

RULE, S. J. Equal discriminability scale of number. Journal of Experimental Psychology, 1969, 79, 35-38.

RULE, S. J. Discriminability scales of number for multiple and fractional estimates. Acta Psychologica, 1971, in press.

RULE, S. J., CURTIS, D. W. \& MARKLEY, R. P. Input and output transformations from magnitude estimation. Journal of Experimental Psychology, 1970, 86, 343-349.

(Received for publication April 7, 1971.) 